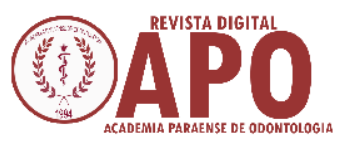

\title{
Terceiro molar ectópico em côndilo associado a um cisto dentígero: relato de caso e revisão de literatura
}

Ectopic third molar in mandibular condyle associated with dentigerous cyst: a case report and literature review

\author{
Aline Marques Dias ${ }^{1}$ \\ Beatriz Voss Martins ${ }^{2}$ \\ Fernando Jordão de Sousa Junior ${ }^{1}$ \\ Luiz Jorge de Araújo Guedes ${ }^{3}$ \\ Maria Sueli da Silva Kataoka ${ }^{4}$
}

\section{RESUMO}

Terceiros molares localizados no côndilo mandibular são considerados achados raros e poucos foram relatados na literatura. O objetivo deste trabalho foi relatar um caso incomum de terceiro molar ectópico em côndilo, associado a um cisto dentígero, bem como a abordagem cirúrgica utilizada e as características da região afetada através de exames de imagem.

Palavras-chave: Côndilo mandibular. Erupção ectópica de dente. Dente impactado. Terceiro molar.

\begin{abstract}
Third molars located in the mandibular condyle are considered rare findings and few cases have been reported in the literature. The aim of this study was to report an unusual case of ectopic third molar in condyle associated with dentigerous cyst, surgical approach used and its characteristics through imaging tests.
\end{abstract}

Keyword: Mandibular condyle. Ectopic tooth eruption. Impacted tooth. Third molar.

\footnotetext{
${ }^{1}$ Mestre em Odontologia, Universidade Federal do Pará

${ }^{2}$ Graduanda, Faculdade de Odontologia, Universidade Federal do Pará

${ }^{3}$ Especialista em Cirurgia Bucomaxilofacial, Associação Brasileira de Ensino Odontológico - SP

${ }^{4}$ Professora Doutora, Faculdade de Odontologia, Universidade Federal do Pará
} 


\section{INTRODUÇÃo}

Terceiros molares não irrompidos são encontrados, em geral, em suas regiões de irrupção usuais ou próximas a elas, porém, ocasionalmente, estes dentes podem ser observados em locais mais distantes da posição normal no arco dentário ${ }^{1}$. A etiologia e o mecanismo da migração dentária intraóssea não são completamente compreendidos, apesar de haver diferentes teorias relacionadas às alterações durante o crescimento mandibular, à presença de patologias associadas, traumas e fatores genéticos.

Terceiros molares mandibulares ectópicos são incomuns, podendo ocorrer em côndilo mandibular ${ }^{2,3,4,5}$, região subcondilar ${ }^{1,7,8,9,10,11,12,13,14,15,16,17}$, ramo da mandíbula ${ }^{18,19}$ ângulo da mandíbula ${ }^{7}$ e processo coronoide $^{20}$. A maioria dos relatos na literatura consta de terceiros molares na região subcondilar, enquanto raramente são observados no côndilo propriamente dito.

É considerável o número de pacientes assintomáticos, de modo que os dentes ectópicos são detectados por achados radiográficos de rotina e, em muitos casos, não é necessário tratamento, a não ser que apresentem sintomatologia ou lesão associada. Quando há relatos de sintomas, estes estão relacionados à cefaleia e dor na articulação temporomandibular ${ }^{2,7}$.

Os dentes ectópicos em côndilo são de difícil remoção, devido à sua localização. A abordagem cirúrgica pode ser realizada por acesso intra ou extraoral e com o auxílio de endoscópio, dependendo da localização, posição do dente e presença de lesões associadas $^{3,6,8}$.

O objetivo deste artigo foi o de realizar uma revisão da literatura acerca de terceiros molares ectópicos em côndilo mandibular e relatar um caso clínico, suas características clínicas e radiográficas, assim como a abordagem cirúrgica de escolha.

\section{REVISÃo DE LITERATURA}

As buscas foram feitas nas bases de dados PubMed, Lilacs e Medline, com as palavras-chave: "mandibular condyle", "ectopic tooth eruption", "impacted tooth" e "third molar", no período de 1970 a 2018. O fator de inclusão foi a localização do terceiro molar ectópico no côndilo mandibular, e o de exclusão, foi a localização em outras regiões. Foram identificados 21 artigos relatando terceiros molares inferiores ectópicos. Destes, apenas 4 estavam no interior do côndilo (Quadro 1), 10 estavam localizados na região subcondilar, e 7 estavam em outras regiões tais como: ângulo e ramo da mandíbula, apófise coronoide e espaço pterigomandibular. Portanto, o caso relatado abaixo representa o quinto paciente com terceiro molar ectópico, em côndilo.

Quadro 1. Relatos na literatura de terceiro molar ectópico em côndilo, em ordem cronológica

\begin{tabular}{|l|c|c|l|c|}
\hline \multicolumn{1}{|c|}{ Autor/ano } & Idade & Sexo & \multicolumn{1}{|c|}{ Sinais e Sintomas } & $\begin{array}{c}\text { Tipo de } \\
\text { tratamento }\end{array}$ \\
\hline $\begin{array}{l}\text { Suarez-Cunqueiro } \\
\text { et al., 2003 }\end{array}$ & 45 & $\mathrm{M}$ & Dor e edema no lado direito da face & $\begin{array}{c}\text { Cirúrgico, } \\
\text { via intraoral } \\
\text { endoscópica }\end{array}$ \\
\hline Gadre et al., 2009 & 30 & $\mathrm{~F}$ & $\begin{array}{l}\text { Dor e edema na região pré-auricular } \\
\text { direita }\end{array}$ & $\begin{array}{c}\text { Cirúrgico, } \\
\text { via intraoral }\end{array}$ \\
\hline Kim, 2011 & 70 & $\mathrm{~F}$ & $\begin{array}{l}\text { Desconforto na região pré-auricular } \\
\text { esquerda durante a realização dos } \\
\text { movimentos mandibulares }\end{array}$ & Conservador \\
\hline Lambade et al., 2013 & 35 & $\mathrm{~F}$ & $\begin{array}{l}\text { Dor na região abaixo da orelha } \\
\text { esquerda e dificuldade para realizar } \\
\text { o movimento de abertura da boca }\end{array}$ & $\begin{array}{l}\text { Cirúrgico, } \\
\text { via extraoral }\end{array}$ \\
\hline Fonte: Pesquisa bibliográfica (Período entre 1970 e 2018). M= Masculino; F= Feminino
\end{tabular}

\section{Relato de CASO}

Paciente de 61 anos, do sexo feminino, apresentouse no Hospital Ophir Loyola, Belém, Pará, queixando-se de dor e inchaço no lado direito da face.

Ao exame físico extraoral verificou-se edema e fístula na região pré-auricular direita (Figura 1), além de dificuldade para realizar os movimentos mandibulares.

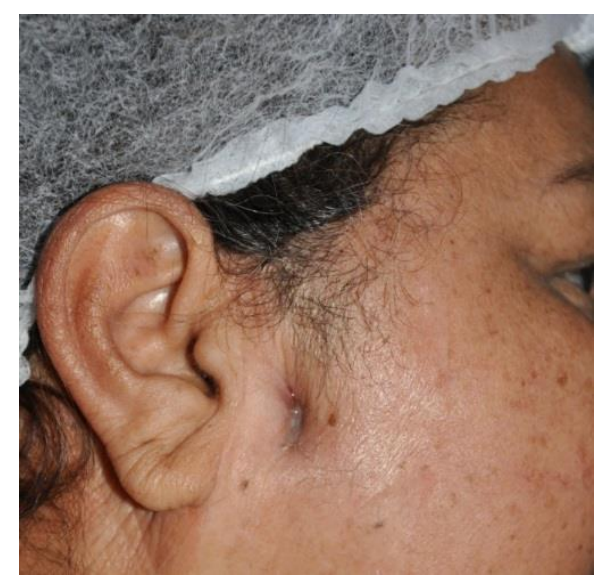

Figura 1. Imagem da área de drenagem purulenta do edema, na região pré-auricular do lado direito. 
Ao exame físico intraoral não foi verificada patologia significativa associada a dentes ou tecidos bucais.

A radiografia panorâmica revelou a presença de imagem compatível com terceiro molar ectópico, na região do côndilo mandibular direito, em posição vertical invertida e presença de imagem radiolúcida de limites definidos e corticalizados envolvendo a coroa do molar, sugerindo cisto dentígero (Figura 2).

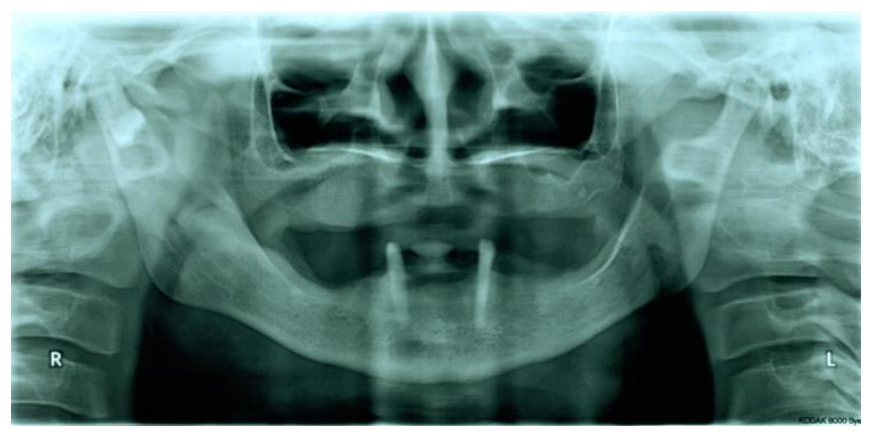

Figura 2. Radiografia panorâmica exibindo terceiro molar mandibular ectópico, localizado no côndilo do lado direito. A coroa do elemento dentário apresenta associação com imagem radiolúcida de limites definidos e corticalizados, sugestiva de cisto dentígero.

Foi realizada a drenagem do conteúdo seropurulento e a instalação de um dreno de penrose (Cirúrgica Brasil, São Paulo, SP, BR). A paciente foi encaminhada para execução de tomografia computadorizada espiral para o planejamento cirúrgico.

Na tomografia pode-se observar a localização do terceiro molar ocupando o interior do côndilo, tendo sua coroa voltada para o colo da cabeça da mandíbula e imagem hipodensa envolvendo a coroa com perda de continuidade na cortical da porção lateral do côndilo, coincidindo com a localização da fístula (Figura 3).

A remoção do dente foi realizada sob anestesia geral e a abordagem cirúrgica foi extraoral (Figura 4), com incisão retromandibular, a fim de obter acesso adequado tanto ao dente ectópico quanto ao cisto dentígero.

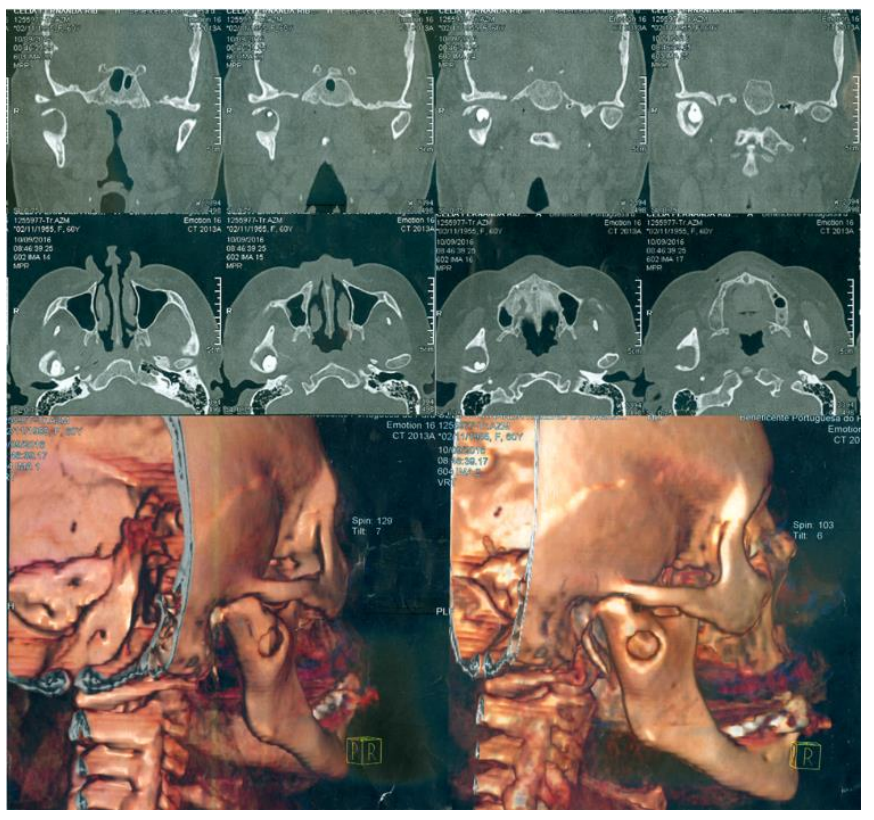

Figura 3. Imagens de tomografia computadorizada espiral, com cortes coronais, axiais e reconstrução em $3 \mathrm{D}$, respectivamente. Observa-se a localização do terceiro molar mandibular ectópico no côndilo da mandíbula do lado direito, com imagem hipodensa associada ao elemento dental e perda de continuidade da cortical do processo condilar, em sua porção lateral.

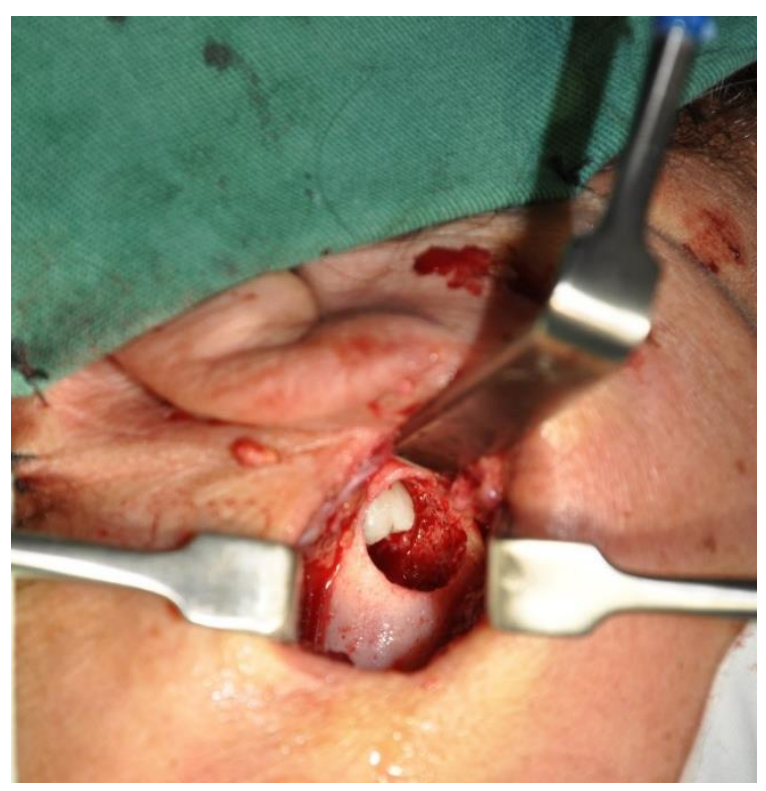

Figura 4. Intervenção cirúrgica na região do côndilo do lado direito, onde é possível observar a coroa do terceiro molar mandibular ectópico. 
Para a remoção do dente foi utilizada broca reta tronco-cônica n ${ }^{\circ} 702$ (KG Sorensen, São Paulo, SP, BR) para osteotomia e odontossecção. Foi realizada, também, a enucleação da lesão, cuja hipótese de cisto dentígero foi confirmada posteriormente por exame histopatológico.

A paciente não apresentou intercorrência durante o procedimento ou no pós-operatório, nem lesões ao nervo facial, sendo orientada a retornar após seis meses para reavaliação.

\section{DiscuSSão}

É considerado um dente ectópico aquele que é encontrado distante de sua localização anatômica, em posição anômala ou fora do seu eixo oclusal no arco dentário ${ }^{2,420}$. A etiologia e o mecanismo da migração dentária intraóssea não são completamente compreendidos, apesar de haver algumas teorias. Durante o desenvolvimento da mandíbula, conforme o ramo se alonga e cresce longitudinalmente, o germe do dente pode seguir junto. $\mathrm{O}$ desenvolvimento de um cisto ou tumor associado, como o cisto dentígero, pode pressionar o dente para uma posição anormal, conforme seu crescimento. Além disso, outras possíveis causas para o deslocamento incluem falta de espaço e trauma ${ }^{2}$.

O terceiro molar mandibular é frequentemente encontrado impactado e pode ser classificado de acordo com sua posição anteroposterior entre o segundo molar e o ramo ascendente da mandíbula, sua posição mediallateral no corpo da mandíbula e enfim, segundo seu longo eixo ${ }^{13,18}$. Terceiros molares ectópicos, contudo, são achados raros e descobertos por exames de rotina e somente são tratados quando o paciente apresenta sintomatologia ou lesão associada ${ }^{2,16}$.

A remoção dos terceiros molares da região condilar é recomendado para prevenir infecções e cistos, bem como para diminuir risco de fraturas patológicas e disfunção da articulação temporomandibular ${ }^{18}$.

$\mathrm{Na}$ literatura, entre os quatro casos de terceiro molar ectópico em côndilo, três deles apresentavam lesão cística associada ${ }^{3,4,5}$ e em um caso, houve associação com osteomielite ${ }^{7}$. No caso aqui relatado, o terceiro molar ectópico também estava associado à lesão cística.

Em razão da localização do terceiro molar no côndilo, sua remoção deve ser bem planejada com base na relação anatômica com estruturas nobres, e possíveis sequelas que esta pode causar ao paciente. Existem várias abordagens descritas na literatura, tais como, intraoral, extraoral e endoscópica. A eleição da abordagem cirúrgica está associada à técnica e experiência do cirurgião $0^{7,4,11}$.

Sempre que possível, o tratamento intraoral é adotado $^{12}$ a fim de evitar danos ao nervo facial e cicatrizes ${ }^{13}$. Entretanto, o campo cirúrgico limitado e a pouca visualização são desvantagens desse tipo de técnica cirúrgica ${ }^{6}$. Os acessos extraorais proporcionam uma boa exposição cirúrgica, contudo, são mais suscetíveis a lesões do nervo facial e cicatrizes. Ressaltese que esse tipo de abordagem é mais frequente na literatura, quando o terceiro molar localiza-se nas regiões subcondilar e condilar ${ }^{14,15}$. A técnica endoscópica possui algumas vantagens, como a boa iluminação do campo, porém, é menos utilizada por exigir equipamentos específicos e treinamento para seu manuseio $^{4,14}$.

Neste caso, a escolha da abordagem cirúrgica foi a extraoral, com acesso retromandibular, pela posição do dente, da lesão, e do rompimento da cortical, o que permitiu realizar a osteotomia condilar objetivando alcançar a cortical interna do côndilo e remover tanto o terceiro molar como o cisto associado. Uma abordagem intraoral, por exemplo, resultaria em maior perda óssea, aumentando o risco de fratura. É sempre preferível que o acesso seja o mais conservador possível na região condilar, e quando feita por um cirurgião experiente, os riscos de injúria às estruturas nervosas se tornam mínimos.

Não houve complicações durante o procedimento ou no pós-operatório. A proservação é necessária por um período pós-cirúrgico para acompanhar a completa ossificação da região, por existir risco de fratura, pelo enfraquecimento devido ao trauma cirúrgico. 


\section{Conclusão}

Terceiros molares ectópicos são raros e muitas vezes assintomáticos, sendo descobertos eventualmente em exames de rotina. Quando estes provocam sintomatologia dolorosa e se encontram associados a lesões, o tratamento cirúrgico é indicado. A definição da abordagem cirúrgica deve ser feita através de estudo clínico e exames de imagem preconizando o sucesso do tratamento, manutenção da estética e função das estruturas craniofaciais.

\section{REFERÊNCIAS}

1. Oikarinen VJ, Altonen M. Impacted third molar in condyloid process. Report of a case. Oral Surg. 1970; 30(1):7-10

2. Ahmed NM, Speculand B. Removal of ectopic mandibular third molar teeth: literature review and a report of three cases. Oral Surg. 2012; 5:39-44.

3. Gadre KS, Waknis P. Intra-oral removal of ectopic third molar in the mandibular condyle. Int J Oral Maxillofac Surg. 2010; 39: 292-307.

4. Suarez-Cunqueiro MM, Schoen R, Schramm A, Gellrich NC, Schmelzeisen R. Endoscopic approach to removal of an ectopic mandibular third molar. $\mathrm{Br} \mathrm{J}$ Oral Maxillofac Surg. 2003; 41:340-2.

5. Kim J. Cone beam computed tomography findings of ectopic mandibular third molar in the mandibular condyle: report of a case. Imaging Sci Dent. 2011; 41:135-7.

6. Iglesias-Martins F, Infante-Cossio P, Carranza ET, Prats-Golczer VE, Garcia-Perla-Garcia A. Ectopic third molar in the mandibular condyle: A review of literature. Med Oral Patol Cir Bucal 2012 nov;17(6) e1013-7.

7. Lambade P, Lambade D, Dolas RS, Virani N. Ectopic mandibular third molar leading to osteomyelitis of condyle: a case report with literature review. Oral Maxillofac Surg. 2013; 17:127-30.

8. Goyal AK, Gupta DS, Jain R. Intraoral removal of bilateral ectopic third molar in the subcondylar and ramus regions: a rare case report. J Stomat Occ Med. 2012; 5:94-6.

9. Bortoluzzi MC, Manfro R. Treatment for ectopic third molar in the subcondylar region planned with cone beam computed tomography: A case report. J Oral Maxillofac Surg. 2010; 68:870-2.

10. Bux P, Lisco V. Ectopic third molar associated with a dentigerous cyst in the subcondylar region: report of case. J Oral Maxillofac Surg. 1994; 52:630-2.
11. Bowman J, O'Regan B, Bhopal S. Transmasseteric antero-parotid approach: a technique adaptation for ectopic subcondylar third molar removal and associated dentigerous cyst enucleation. Br J Oral Maxillofac Surg. 2014; 52, e7-e8.

12. Wassouf A, Eyrich G, Lebeda R, Grätz KW. Surgical removal of a dislocated lower third molar from the condyle region: Case report. Schweiz Monatsschr Zahnmed. 2003; 113(4):417-20

13. Lee Y, Park S, Myoung H. Surgical extraction of mandibular third molar in pterygomandibular space: a case report. J Korean Assoc Oral Maxillofac Surg. 2013; 39:242-5.

14. Salmeron JI, del Amo A, Plasencia J, Pujol R, Vila CN. Ectopic third molar in condylar region. Int J Oral Maxillofac Surg. 2008; 37: 398-400.

15. Pace C, Holt D, Payne M. An unusual presentation of an ectopic third molar in the condylar region. Aust Dent $J$. 2010; 55:325-7.

16. Tümer C, Eset AE, Atabek A. Ectopic impacted mandibular third molar in the subcondylar region associated with a dentigerous cyst: A case report. Quintessence Int. 2002; 33:231-3.

17. Shivashankara C, Manjunatha BS, Tanveer A. Ectopic mandibular third molar in subcondylar region: report of a rare case. Oral Maxillofac Surg. 2012; 16:153-5.

18. Wang C, Kok S, Hou L, Yang P, Lee J, Chang S, et al. Ectopic mandibular third molar in the ramus region: report of a case and literature review. Oral Surg Oral Med Oral Pathol Oral Radiol Endod. 2008; 105:155-61.

19. McCrea S. Adjacent dentigerous cysts with the ectopic displacement of a third mandibular molar and supernumerary (forth) molar: a rare occurrence. Oral Surg Oral Med Oral Pathol Oral Radiol Endod. 2009; 107: e15-e2.

20. Martorelli SBF, Albuquerque RS, Marinho EVS, Coelho-Júnior EC, Martorelli FO, Melo JF. Inclusões ectópicas em apófise coronoide. Relato de casos geneticamente associados. Ectopic inclusions in coronoid apophysis: report of cases with familial association. Rev Cir Traumatol Buco Maxilofac. 2007; $7(3): 41-8$. 